\title{
Análise dos motivos para a prática de indivíduos com deficiência em modalidades coletivas e individuais
}

\author{
Analysis of motives for practice of individuals with disability \\ in individual and group sports
}

\author{
Marcus Vinícius Mizoguchi ${ }^{1}$ \\ Francieli Santos Mandarino² \\ Weslei Jacob² \\ Luciana Boligon Refundini²
}

\section{RESUMO}

O objetivo do presente trabalho foi analisar os motivos para prática de indivíduos com deficiência física e visual em modalidades coletivas e individuais. A presente pesquisa trata de um estudo descritivo, sendo realizado com 30 pessoas com alguma deficiência física ou visual (adquirida ou congênita), com aplicação do questionário Exercise Motivation Inventory - (EMI-2), para avaliar os motivos para a prática esportiva. Para análise de dados foram utilizados os testes Shapiro-wilk, Anova de medidas repetidas e U de Mann-whitney, adotando $p<0,05$. Em relação aos níveis dos motivos para prática de exercício físico de indivíduos com deficiência física em modalidades coletivas e individuais, houveram prevalências nas dimensões de prevenção de doenças, condição física, controle de estresse, diversão/bem-estar, afiliação e competição. Na comparação dos motivos para a prática frente às diferentes deficiências não houve diferença significativa importante. Por fim, houve uma diferença significativa entre os menos experientes e os mais experientes, destacando a dimensão de prevenção de doenças para os iniciantes. Pode-se concluir que indivíduos com deficiência física e visual buscam a prática de uma modalidade esportiva para prevenção de doenças, condição física, controle do estresse, diversão/bem-estar, afiliação e competição.

\section{PALAVRAS-CHAVE:}

Deficiência Física, Deficiência Visual, Motivação.

\footnotetext{
${ }^{1}$ Universidade Federal de Mato Grosso.

2 Faculdade Metropolitana de Maringá.
} 


\section{ABSTRACT}

The aim of the present paper was to analyze the motives for practice of individuals with Physical Disability in individual and group sports. The research is caracterized in a descriptive study. It was conducted with 30 players with some physical disability (acquired or congenital), using the Exercise Motivation Inventory - (EMI2) to analyze the motives in sport practices. For data analysis, we used the Shapiro-wilk test, ANOVA for repeated measure and $U$ Mann-Whitney, adopting $p<0,05$. The results showed that the levels of motives to physical exercise in players with physical disability in sports was highlighted the disease prevention, Physical condition, stress control, fun/well-being, affiliation and competition. Comparing the reasons for the practice between different disabilities, there was no major significant difference. Finally, there was a significant difference between less experienced and more experienced, highlighting the disease prevention for the beginners. It concluded that individuals with physical diseases seek disease prevention, physical condition, stress control, fun/well-being, affiliation and competition.

\section{KEYWORDS}

Physical Disability, Visual Disability, Motivation. 


\section{INTRODUÇÃO}

Cerca de 23,9\% da população brasileira declara obter algum tipo de deficiência, subdivididas entre visual, auditiva, motora e mental. Verificou-se que a deficiência visual apresenta maior incidência, representando $18,8 \%$ da população, seguida por deficiência motora (7\%), auditiva (5,1\%), e mental ou intelectual (1,4\%) (IBGE, 2010). Desta maneira, a prática esportiva para pessoas com deficiência tem recebido uma maior atenção, podendo observar uma evolução nos jogos paralímpicos. Este avanço é identificado pelo quadro de medalhas conquistas pelo Brasil nos últimos dois jogos paralímpicos (43 medalhas em 2012 e 72 medalhas em 2016). Com tamanha elevação na participação do público em questão, torna-se fundamental a adaptação de espaços, métodos de trabalho, modificações em regras desportivas dentre outros ajustes para otimizar a inserção de pessoas com algum tipo de deficiência no contexto esportivo (CARDOSO, 2011; GORGATTI; 2005; DUARTE, WERNER, 1995).

Durante anos a literatura buscou realizar estudos voltados às temáticas pautadas nos mecanismos de inclusão e reabilitação de pessoas com alguma deficiência física elou visual (AZEVEDO, BARROS, 2004; MELO, 2004, LABRONICl et al, 2000). Com a inserção deste público na prática esportiva surgiram novos caminhos para possibilitar a interação dessas pessoas com a sociedade, evidenciando as capacidades residuais com a deficiência (GORLA, ARAÚJO, CARMINATO, 2004; ARAÚJO, 1997).

Oliveira et al. (2013) analisaram a motivação entre paratletas com deficiência visual e física que praticam natação e atletismo, e concluíram que praticantes de natação adaptada são mais incisivos na busca da performance e reconhecimento social. Para tanto, existe uma forte lacuna nos achados científicos atuais em compreender os motivos reais pelos quais praticantes de modalidades esportivas coletivas ou individuais optam por adentrar a um programa de treinamento. Desta forma, Hutzler, Oz e Barak (2013) sugerem novos estudos sobre motivação entre deficientes físicos e mentais.

A motivação tem sido um dos fatores mais importantes dentro da Psicologia do Esporte. Ao avaliar as principais temáticas de trabalhos em periódicos e congressos de psicologia do esporte, Vieira, Nascimento Junior e Vieira (2013) verificaram que a motivação é a variável psicológica mais estudada nos últimos 15 anos. Dentro dessa perspectiva, a Teoria da Autodeterminação (DECl; RYAN, 1985) explica que o indivíduo, praticante de qualquer atividade, é motivado por fatores intrínsecos e extrínsecos. Sendo assim, a prática esportiva pode ser praticada por ser algo interessante e prazerosa para a pessoa (intrínseca), ou fazer por ter outros objetivos ou causas externas (extrínsecas) (DECl; RYAN, 2012).

Boas, Bim e Barian (2003) investigaram a motivação de praticantes de basquetebol sobre cadeira de rodas, evidenciando que $50 \%$ dos indivíduos buscaram a atividade com o intuito de lazer e recreação (motivação intrínseca). No entanto, percebeu-se uma melhora na autoestima, relacionamentos pessoais, habilidades motoras e ocupação do tempo livre. Desta forma, percebe-se que a prática de modalidades esportivas oferece uma melhora nos diversos aspectos do praticante.

Dentro dessa perspectiva, inúmeros estudos têm surgido sobre às contribuições que o esporte e ou atividade física podem oferecer às pessoas com limitações físicas. Para Zuchetto e Castro (2002), o esporte preferido de pessoas com alguma deficiência física elou visual é a natação adaptada e o basquete sobre cadeira de rodas, os quais enfatizam os benefícios de realizar atividades devido a melhora da qualidade de vida. Corroborando com essa relação, a melhora da integração social tem trazido inúmeros benefícios orgânicos adquiridos por esportistas com deficiência (COSTA et al, 2014; TSUTUMI et al, 2004). Assim, o esporte adaptado passa a ser parte integrante da sociedade, tornando-se alvo de inúmeras pesquisas, bem como de possibilidades de intervenção profissional e social. O desporto adaptado pode ser definido como esporte modificado ou especialmente criado para ir ao encontro das necessidades únicas de indivíduos com algum tipo de deficiência (GORGATTI; GORGATTI, 2005).

Desta forma, o objetivo do presente estudo foi analisar os motivos para prática de indivíduos com deficiência física e visual em modalidades coletivas e individuais. Como objetivos específicos buscaram-se: 
a) Identificar os motivos da prática esportiva em atletas com deficiência física e visual, b) comparar os motivos para prática entre as diferentes deficiências (adquirido e congênito) e c) comparar os motivos de acordo com o tempo de prática (menos experientes e mais experientes).

\section{MÉTODOS}

\section{População e amostra}

A população investigada foi um grupo de atletas com deficiência física e visual, exceto indivíduos com deficiência cognitiva, sendo de modalidades individuais e coletivas da cidade de Maringá - PR. Para amostra do estudo foi investigado 30 atletas de 15 a 50 anos das modalidades de basquete em cadeira de rodas, vôlei sentado, atletismo adaptada e natação adaptada (Tabela 1). Os critérios de inclusão para participar desta pesquisa: a) ter algum tipo de deficiência física e visual (deficiência congênita ou adquirida), b) estarem cadastrados em alguma equipe de esporte adaptado da cidade de Maringá. Como critérios de exclusão: a) atletas com qualquer tipo de deficiência cognitivas b) não assinatura do termo de consentimento livre e esclarecido.

Tabela 1. Dados de identificação dos atletas praticantes de modalidades adaptadas.

\begin{tabular}{|c|c|c|c|c|c|c|}
\hline \multirow[b]{2}{*}{ Modalidade } & \multirow[b]{2}{*}{$\begin{array}{l}\text { Idade } \\
(\bar{X} \bar{X} \pm d p)\end{array}$} & \multicolumn{2}{|l|}{ Sexo } & \multicolumn{3}{|c|}{ Te m p o de Tipo de deficiência } \\
\hline & & Masc. & Fem. & Prática $(\bar{X} \bar{X}$ & Adq. & Cong. \\
\hline Natação & $27,9 \pm 13,28$ & 4 & 3 & $3,3 \pm 2,21$ & 5 & 2 \\
\hline Bocha & $34,6 \pm 15,39$ & 6 & 1 & $7,0 \pm 4,16$ & 1 & 6 \\
\hline Goalball & $31,2 \pm 7,08$ & 6 & 0 & $6,8 \pm 2,32$ & 3 & 3 \\
\hline Basquete e Handebol & $03,2 \pm 7,13$ & 10 & 0 & $7,0 \pm 2,45$ & 10 & 0 \\
\hline Total & $31,2 \pm 10,74$ & 26 & 4 & $6,1 \pm 3,14$ & 19 & 11 \\
\hline
\end{tabular}

\section{Instrumentos e protocolos de medida}

Os instrumentos utilizados foram: Ficha de identificação e o questionário Exercise Motivation Inventory - (EMI-2) de Markland e Ingledew (1997), traduzido e adaptado para o idioma português por Guedes, Legnani e Legnani (2011).

Com o intuito de ter as informações dos indivíduos, foi entregue a ficha de identificação, contendo os dados pessoais como telefone, email, endereço e escolariedade, idade, tipo de deficiência (congênito ou adquirido) e qual deficiência (visual ou físico); e os dados atléticos como modalidade e tempo de prática. Para avaliar os motivos para prática esportiva, utilizou-se o Exercise Motivation Inventory (EMI-2). Está avaliação é realizada numa escala likert de 0 a 5 pontos, entre "nada/pouco verdadeiro" e "Muito verdadeiro". O questionário consiste em 44 afirmações relacionadas aos motivos para prática, sendo caracterizada por 10 dimensões: a) prevenção de doenças, b) condições físicas, c) controle do peso corporal, d) aparência física, e) controle do estresse, f) diversão/bem-estar, g) afiliação, h) reabilitação da saúde, i) competição, e j) reconhecimento social.

\section{Análise dos dados}

Para a análise dos dados, utilizou-se a estatística descritiva e inferencial. Para identificar a normalidade dos dados, o teste Shapiro-wilk foi realizado, apresentando dados não paramétricos. Desta forma, os dados descritivos foram apresentados em forma de mediana (Md) e intervalos interquartílicos (Q1-Q3).

Para comparação entre as dimensões motivacionais para a prática, foi realizado o teste Anova de medidas repetidas, juntamente com o teste de esfericidade de Mauchy's, Greenhouse Geiser e post-hoc de bonferroni. Na comparação entre as deficiências (congênita e adquirida), entre as modalidades esportivas (individuais e coletivas) e o tempo de prática (Menos experientes e mais experientes), foi feito o teste $U$ de Mann-Whitney, adotando significância de $95 \%$. O tempo de prática foi dicotomizado entre até 02 anos de prática (menos experientes) e mais de 10 anos de prática (mais experientes) 


\section{RESULTADOS E DISCUSSÕES}

Baseando-se no objetivo de analisar os motivos que indivíduos com deficiência física buscam na prática de modalidades coletivas e individuais, primeira- mente será apresentada os motivos para prática de exercício físico dos praticantes de modalidades adaptadas. Em seguida, as comparações dos motivos entre os grupos serão identificados. Desta forma, a figura 1 apresenta os motivos para prática de indivíduos com deficiência física.

Figura 1. Níveis dos motivos para prática de exercício físico de indivíduos com deficiência física em modalidades coletivas e individuais.

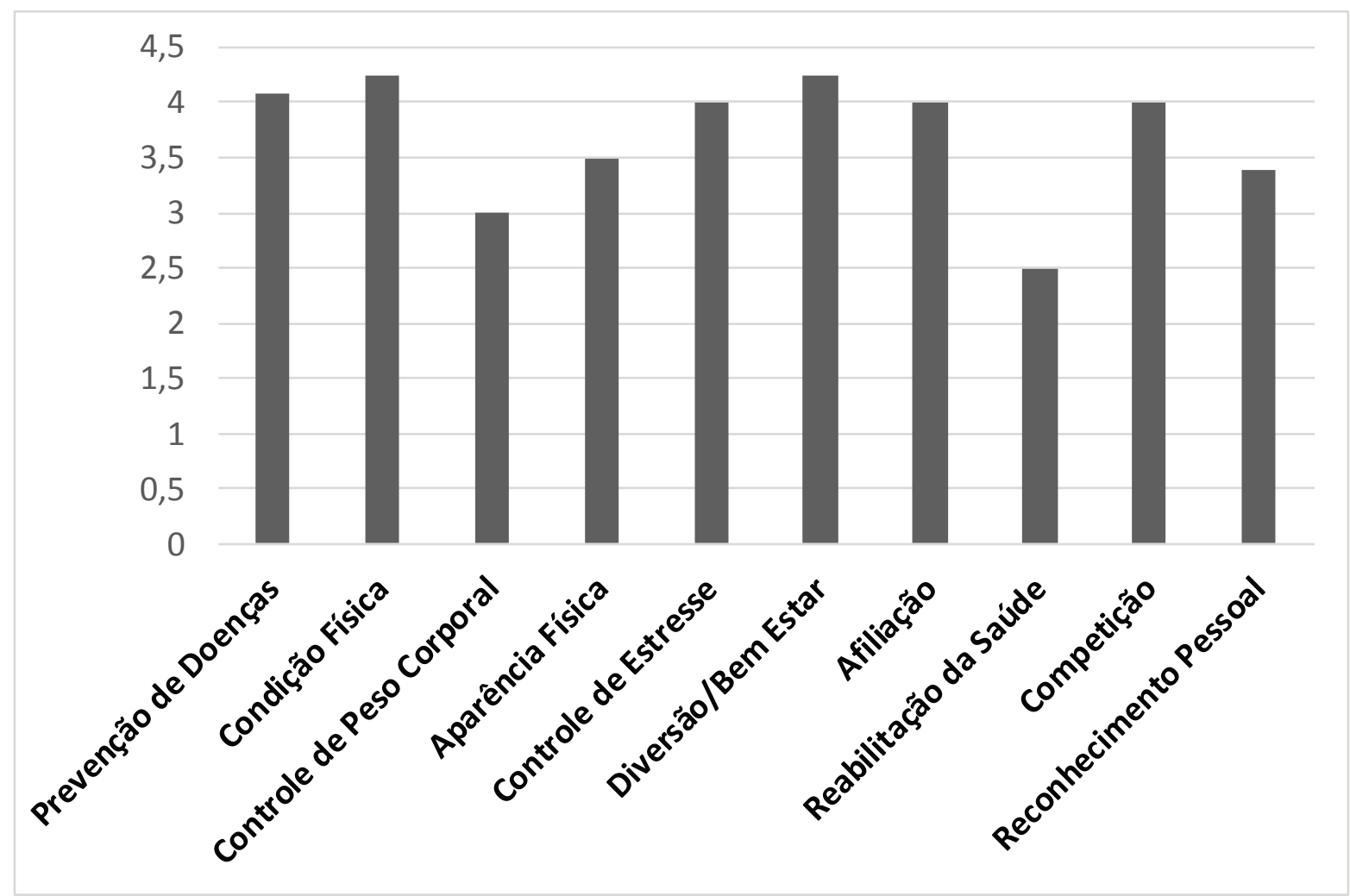

*Diferença significativa $p<0,05$

Conforme os dados acima pode-se observar que existe uma diversidade de motivos para prática de modalidades esportivas com prevalência das condições físicas $(M d=4,25)$, Diversão/bem estar $(\mathrm{Md}=4,25)$ e Prevenção de doenças $(M d=4,08)$. Entretanto, as dimensões de reabilitação da saúde $(\mathrm{Md}=2,50)$ e controle de peso corporal $(\mathrm{Md}=3,00)$ apresentaram menores valores $(p<0,05)$.

Os achados no estudo de Saito (2007) corroboram com os resultados do presente estudo, evidenciando que não existe somente um fator que determina a prática de exercício físico em deficientes físi- cos. Desta forma, foi identificado que orientação para a equipe, a busca de uma boa forma física, estar com os amigos e a realização pessoal foram os motivos mais prevalentes, diferente dos motivos de aquisição de status e descarga de energia. Em contrapartida, Guedes, Legnani e Legnani (2012), demonstraram que a prevenção de doenças foi um dos principais fatores de motivação em universitários praticantes de exercício físico, podendo verificar diferentes metas entre as populações. A tabela 2 apresentou uma comparação dos motivos para prática entre adquiridos e congênitos. 
Tabela 2. Comparação dos motivos para prática frente às diferentes deficiências.

\begin{tabular}{llll}
\hline \multirow{2}{*}{ Dimensão } & Adquirido $(\mathrm{n}=19)$ & \multicolumn{1}{l}{ Congênito $(\mathrm{n}=11)$} & \multirow{2}{*}{$P$} \\
\cline { 2 - 3 } & Md(Q1-Q3) & Md (Q1-Q3) & 0,63 \\
\hline Prevenção de Doenças & $4,00(2,50-4,67)$ & $4,17(3,33-4,67)$ & 0,48 \\
Condição Física & $4,25(3,00-4,75)$ & $3,75(3,00-4,50)$ & 0,96 \\
Controle de Peso Corporal & $3,00(2,00-3,25)$ & $2,75(2,25-3,25)$ & 0,98 \\
Aparência Física & $3,50(2,00-3,75)$ & $3,50(1,75-3,75)$ & 0,26 \\
Controle de Estresse & $4,25(3,50-4,50)$ & $3,75(2,50-4,50)$ & 0,25 \\
Diversão/Bem-Estar & $4,33(3,50-5,00)$ & $3,67(3,50-4,67)$ & 0,07 \\
Afiliação & $4,50(3,25-4,75)$ & $3,50(2,50-4,50)$ & 0,30 \\
Reabilitação da Saúde & $2,67(2,00-3,33)$ & $2,33(1,67-2,67)$ & 0,41 \\
Competição & $4,20(3,40-5,00)$ & $4,00(3,00-4,60)$ & 0,66 \\
Reconhecimento Pessoal & $3,25(2,00-4,25)$ & $3,50(1,75-4,25)$ & \\
\hline
\end{tabular}

*Diferença significativa $\mathrm{p}<0,05$

Ao comparar os motivos para prática entre indivíduos com deficiência adquirida e congênita, não foi possível identificar nenhuma diferença significativa $(p>0,05)$. Segundo Duarte e Werner (1995), indivíduos que nasceram ou adquiriram a deficiência não interferem na prática esportiva atual. No entanto, indivíduos com deficiência adquirida sofreram mudanças em suas vidas, e que essas alterações devem ser assimiladas e necessitam de um determinado tempo para adaptarem (Lehnhard et al., 2012). Ou- tro estudo de Parreiras (2008) verificou que o tipo de deficiência não interfere em outros aspectos psicológicos como a percepção da qualidade de vida.

A tabela 3 apresenta a comparação entre o tempo de prática dividindo em menos experiente e mais experiente. Para realizar essa divisão, atletas com menos de 5 anos de experiências na modalidade se enquadraram como menos experientes, e atletas com mais de 5 anos de experiência como mais experientes.

Tabela 3. Comparação dos motivos para a prática em deficientes físicos e visuais de acordo com o tempo de prática.

\begin{tabular}{|c|c|c|c|}
\hline \multirow[b]{2}{*}{ Dimensão } & Menos experientes & Mais experientes & \multirow[b]{2}{*}{$p$} \\
\hline & Md(Q1-Q3) & Md (Q1-Q3) & \\
\hline Prevenção de Doenças & $4,67(3,83-4,83)$ & $3,83(2,50-4,17)$ & $0,02^{*}$ \\
\hline Condição Física & $4,38(3,56-5,00)$ & $3,88(3,00-4,25)$ & 0,08 \\
\hline Controle de Peso Corporal & $3,00(1,44-4,31)$ & $2,88(2,25-3,25)$ & 0,57 \\
\hline Aparência Física & $3,50(2,13-4,13)$ & $3,00(1,81-3,50)$ & 0,36 \\
\hline Controle de Estresse & $4,38(3,69-4,81)$ & $3,50(3,00-4,44)$ & 0,07 \\
\hline Diversão/Bem-Estar & $4,75(3,63-5,00)$ & $3,92(3,50-4,50)$ & 0,13 \\
\hline Afiliação & $4,25(2,88-4,56)$ & $3,88(3,06-4,50)$ & 0,98 \\
\hline Reabilitação da Saúde & $2,50(2,00-3,33)$ & $2,50(1,42-3,00)$ & 0,69 \\
\hline Competição & $4,70(3,60-5,00)$ & $3,90(3,05-4,20)$ & 0,07 \\
\hline Reconhecimento Pessoal & $3,63(2,19-4,31)$ & $3,38(1,75-3,88)$ & 0,54 \\
\hline
\end{tabular}

*Diferença significativa $\mathrm{p}<0,05$

Observou-se que na tabela 3 houve uma diferença significativa nas dimensões entre os grupos. Os menos experientes destacaram maiores valores na dimensão de prevenção de doenças $(\mathrm{Md}=3,83) \mathrm{em}$ comparação aos mais experientes ( $\mathrm{Md}=4,67)$.

De acordo com a Teoria da Autodeterminação (DECl; RYAN, 2012), indivíduos que vivenciam práti- cas positivas, apresentam motivações mais intrínsecas voltadas ao prazer e a satisfação, fazendo com que motivações extrínsecas (como prevenção de doenças) sejam mais insignificantes. Estudos realizados por Santos e Knijnik (2006), Lopes e Nunomura (2007) demonstram que quanto maior o tempo de permanência em um determinado contexto maior é o nível de prazer e gosto pela prática. 


\section{CONCLUSÃO}

O trabalho buscou analisar os motivos para prática de indivíduos com deficiência física em modalidades coletivas e individuais. Ficou evidente que existem diversos motivos que levam indivíduos com deficiência física e visual a buscar a prática de modalidades esportivas, prevalecendo às condições físicas, diversão, bem estar e prevenção de doenças.

Em relação as comparação dos motivos para prática entre deficiências não houve resultados significantes. Enquanto na comparação frente ao tempo de prática, pode-se observar maiores motivações para prevenção de doenças em indivíduos menos experientes.

Como limitação do presente trabalho, houve dificuldade em coletar números representativos de atletas nas modalidades adaptadas, devido número reduzido de praticantes. Para futuros estudos, deve-se analisar a questão financeira nas modalidades adaptadas, pois estas modalidades esportivas são de alto custo e esses indivíduos não recebem ajuda financeira para a prática das mesmas.

\section{RERERÊNCIAS}

ARAÚJO, P. F. Desporto Adaptado no Brasil: origem, institucionalização e atualidades. 1997. 140f. Tese (Doutorado) Faculdade de Educação Física, Universidade Estadual de Campinas, Campinas, 1997.

AZEVEDO, Paulo H.; BARROS, Jônatas de F. O nível de participação do Estado na gestão do esporte brasileiro como fator de inclusão social de pessoas portadoras de deficiência. Brasília: Revista Brasileira de Ciências e Movimento, 2004.

BOAS, M.S.V.; BIM, R.H.; BARIAN, S.H.S. Aspectos motivacionais e benefícios da prática do basquetebol sobre rodas. Revista da Educação Física/UEM, v. 14, n.02, p. 7-11, 2003.

CARDOSO, Vinicius D. A reabilitação de pessoas com deficiência através do desporto adaptado. Florianópolis, SC: Revista Brasileira de Ciências e Esporte, v.33, n. 2, 2011. p.829-839.

COSTA, L. C. A., et al. O sentido do esporte para atletas de basquete em cadeiras de rodas: processo de integração social e promoção de saúde. Revista Brasileira de Ciências do Esporte, Florianópolis, v. 36, n. 1, p. 123-140, 2014.
DUARTE, E.; WERNER, T. Conhecendo um pouco mais sobre as deficiências. In: Curso de atividade física e desportiva para pessoas portadoras de deficiência: educação à distância. Rio de Janeiro: UGF, v.3, 1995.

GORGATTI, M. G.; GORGATTI, T. O esporte para pessoas com necessidades especiais. In GORGATTI, M. G.; COSTA, R. F. (Org.), Atividade física adaptada: qualidade de vida para pessoas com necessidades especiais. Barueri: Manole, 2005. p. 532-568.

GORLA, J. I.; ARAÚJO, P. F.; CARMINATO, R. A. Desempenho psicomotor em portadores de deficiência mental: avaliação e intervenção. Revista Brasileira de Ciências do Esporte/ Campinas, v. 25, n. 3, p. 133-147, 2004.

GUEDES, D.P.; LEGNANI R.F.S.; LEGNANI E. Propriedades psicométricas da versão brasileira do Exercise Motivations Inventory (EMI-2). Motriz, v.18, n.4, p. 667-677, 2011.

HUTZLER, Y.; OZ, M.; BARAK, S. Goal perspectives and sport participation motivation of Special Olympians and typically developing athletes. Research in Developmental Disabilities, v. 34, n. 07, p. 2149-2160, 2013.

LEHNHARD, G.R.; MANTA, S.W.; PALMA, L.E. A prática de atividade física na história de vida de pessoas com deficiência física. Revista da Educação Física, v. 23, n.1, p. 45$56,2012$.

LABRONICI, R. H. D. D., et al. Esporte como fator de integração do deficiente físico na sociedade. Arquivo de Neuro-Psiquiatria/São Paulo, v. 58 n. 4. 2000.

LOPES, P.; NUNOMURA, M. Motivação para a prática e permanência na ginástica artística de alto nível. Revista Brasileira de Educação Física, v. 21, n. 3., p. 177-187, 2007.

MARKLAND, D.; INGLEDEW, D.K. The measurement of exercise motives: factorial validity and invariance across gender of a revised Exercise Motivations Inventory. British Journal of Health Psychology, v.2, p.361-376, 1997.

OLIVEIRA, G. T. A., et al. Fatores motivacionais em paratletas com deficiência visual e motora praticantes de natação e atletismo. Revista da Faculdade de Educação Física da UNICAMP/Campinas, v. 11, n. 1, p. 29-41, 2013.

PARREIRAS, L.A.M. Análise dos fatores que influenciam a qualidade de vida de atletas paraolímpicos em ambientes de treino e competição. Dissertação (Mestrado) - Universidade Federal de Minas Gerais, Escola de Educação Física, Fisioterapia e Terapia Ocupacional, 2008

SAITO, S.P.D. Motivos que levam indivíduos portadores de deficiência visual a participarem de atividades desportivas adaptadas de alto nível. Revista Mineira de Educação Física, v. 15, n.2, p. 20-32, 2007. 
SANTOS, S.C.; KNIJNIK, J.D. Motivos de adesão à prática de atividade física na vida adulta intermediária1 adulta intermediária1. Revista Mackenzie de Educação Física e Esporte, v. 5, n.1, p. 23-34, 2006.

MELO, J. P. O ensino da educação física para deficientes visuais. Revista Brasileira de Ciências do Esporte/Campinas, v. 25, n. 3, p. 117-131, 2004.
ZUCHETTO, A. T., CASTRO, R. L. V. G. As contribuições das atividades físicas para a qualidade de vida dos deficientes físicos. Revista Kinesis, Santa Maria, n. 26, p. 52-166, 2002. 